\title{
GERMINAÇÃO E CRESCIMENTO DE CALOPHYLLUM BRASILIENSE (CLUSIACEAE), UMA ESPÉCIE TÍPICA DE FLORESTAS INUNDADAS ${ }^{1}$
}

\author{
Márcia C. M. Marques ${ }^{2}$ \\ Carlos A. Joly ${ }^{3}$
}

Recebido em 24/06/1999. Aceito em 26/01/2000

\begin{abstract}
RESUMO - (Germinação e crescimento de Calophyllum brasiliense (Clusiaceae), uma espécie típica de florestas inundadas). A saturação hídrica do solo e a conseqüente diminuição na quantidade de oxigênio disponível para o sistema radicular selecionaram, ao longo do processo evolutivo, plantas capazes de sobreviverem à inundação sazonal ou permanente. Nas espécies neotropicais as adaptações que permitem suportar este estresse são bastante diversificadas. Neste trabalho foram estudados a germinação das sementes e o crescimento inicial das plantas de Calophyllum brasiliense, em condições de hipoxia. As sementes de $C$. brasiliense não são fotoblásticas, sobrevivem ao menos três meses submersas, mas germinam apenas em solo bem drenado. A inundação não inibe o crescimento da planta. Não houve mudanças morfológicas na parte aérea das plantas, mas a substituição das raízes formadas no período pré-inundação por um sistema radicular eficiente talvez seja o principal motivo que permita o crescimento normal das plantas em áreas inundadas. Ao contrário de algumas espécies de locais sujeitos ao alagamento, cujas plantas têm o crescimento inibido pelo estresse, o ciclo de vida de $C$. brasiliense está adaptado à inundação temporária ou permanente. A diversificação da forma de dispersão, a indiferença em relação à luz e a capacidade de sobreviver e crescer em solo inundado são características que contribuem para a ampla distribuição geográfica da espécie, sempre associada a áreas alagáveis.
\end{abstract}

Palavras-chave - arbórea, neotropical, germinação, hipoxia, tolerância à inundação

ABSTRACT - (Seed germination and growth of Calophyllum brasiliense (Clusiaceae), a typical species of flooded forests). Soil waterlogging and the subsequent reduction in the amount of oxygen available for the respiration of the root system selected, along the evolutive process, plants able to thrive in seasonally or permanently flooded areas. In neotropical plants there are many types of adaptations to flooding. In this paper we present the results of the work carried out with seeds and seedlings of $C$. brasiliense subjected to hypoxia during germination and early development. $C$. brasiliense seeds are not photoblastic and survive up to three months burried in a water saturated substrate, but germination only takes place in well-drained soils. Soil waterlogging does not inhibit seedling growth and there are no apparent morphological changes

Parte da Dissertação de Mestrado da primeira Autora

Departamento de Botânica, SCB, Universidade Federal do Paraná, C. Postal 19031, CEP 81531-970, Curitiba, PR, Brasil, e-mail: mmarques@bio.ufpr.br, autor para correspondência

3 Departamento de Botânica, IB, Universidade Estadual de Campinas, C. Postal 6109, CEP 13083-970, Campinas, SP, Brasil 
of the aerial part of flooded plants. New and aerated roots that make plant survival possible replace old and spoiled roots. In contrast to many typical species of flood-prone areas where growth is inhibited by oxygen stress. C. brasiliense seedlings seem to be well adapted to their waterlogged environment. Seed dispersion, the absence of photoblastic response as well as seed and seedling capacity of surviving and growing in waterlogged soils contribute to the wide geographic distribution of $C$. brasiliense always associated with areas subjected to soil waterlogging.

Key words - flooding tolerance, germination, hypoxia, neotropical

\section{Introdução}

A inundação desencadeia uma série de processos físico-químicos e biológicos que influenciam na qualidade do solo como meio para o desenvolvimento das plantas (Ponnamperuma 1984). Nestas condições, tanto a germinação das sementes quanto o crescimento das plantas podem ser comprometidos, dependendo da espécie em questão e da duração da inundação (Kozlowski 1984). Para sobreviver nesta situação as plantas podem apresentar adaptações morfológicas, anatômicas ou metabólicas, de forma a minimizar os efeitos da falta de oxigênio (Hook 1984; Joly 1991; Crawford \& Brändle 1996).

Ambientes florestais neotropicais sujeitos à inundação variam muito em relação à duração, à freqüência e à amplitude do alagamento. Nas várzeas e igapós amazônicos, por exemplo, a inundação é sazonal e chega a cobrir as copas das árvores durante aproximadamente seis meses. Nas florestas da planície litorânea, bastante comuns no sudeste e sul do Brasil, a inundação pode ser permanente ou periódica, atingindo alguns centímetros acima do nível do solo (Scarano 1998). Em florestas ciliares, a inundação varia desde sazonal, nas florestas de galeria, até o alagamento permanente das florestas higrófilas, refletindo na composição florística e estrutura destes tipos vegetacionais (Rodrigues 1989). Espécies de ambientes permanentemente inundados ou desenvolveram adaptações que lhes permitem se estabelecer e sobreviver na ausência de oxigênio, ou necessitam aproveitar os curtos e imprevisíveis períodos em que o ní- vel de água recua, disponibilizando pequenas manchas de solo bem drenado para a germinação ou desenvolvimento da planta.

Entre as plantas típicas de ambientes inundáveis, Calophyllum brasiliense Camb. é uma das espécies arbóreas de maior distribuição, ocorrendo desde a América Central até o litoral sul do Brasil, em Floresta Amazônica, Floresta Atlântica e de Restinga e em Florestas de Galeria e Ciliares do interior do país, principalmente em locais com solo inundado (Reitz et al. 1978; Oliveira-Filho \& Ratter 1995). Embora ainda freqüentes, em algumas localidades as populações de $C$. brasiliense estão sofrendo forte pressão, seja pela exploração ilegal de madeira, como nas florestas da planície do litoral norte do Estado do Paraná (R. X. de Lima, Sociedade de Pesquisa da Vida Selvagem e Educação Ambiental, comunicação pessoal) ou pela destruição da floresta ciliar, que ocorre principalmente no interior do Estado de São Paulo, devido ao avanço das áreas agrícolas (Salvador 1987).

No presente trabalho foram estudados experimentalmente a germinação das sementes e o crescimento de plântulas de $C$. brasiliense em condições de hipoxia, a fim de entender os mecanismos básicos de sobrevivência da espécie nestas condições e contribuir para o conhecimento das estratégias adaptativas de plantas neotropicais à inundação. As informações ecofisiológicas geradas também serão úteis para trabalhos de silvicultura, podendo servir como subsídio para projetos de recomposição de áreas degradadas. 


\section{Material e métodos}

As sementes de $C$. brasiliense utilizadas foram coletadas em diferentes matrizes de uma floresta higrófila, no município de Brotas, SP, em julho/1991, para os experimentos de germinação, e, em maio/1991, para os experimentos com plântulas. Em todos os casos foram removidos do fruto, o epicarpo e o mesocarpo, ficando o endocarpo aderido à testa, à semelhança do que se observa nas sementes encontradas no solo das florestas.

Testes preliminares mostraram que a germinação em papel filtro é muito inferior à observada em areia, motivo pelo qual todos os experimentos foram conduzidos neste substrato. Considerou-se a protrusão da radícula como indicativo de germinação. Os experimentos foram realizados no Laboratório de Ecofisiologia do Departamento de Botânica da UNICAMP e no viveiro de mudas da Prefeitura Municipal de Brotas, SP.

Germinação em diferentes condições de luz - Sementes foram esterilizadas em hipoclorito de sódio a $1 \%$ e divididas em dois grupos de 100 sementes cada. O primeiro foi acondicionado em cinco placas de Gerbox contendo areia peneirada, lavada em água destilada e esterilizada, mantido em germinador B.O.D. (Fanem147) à temperatura de $25 \pm 2^{\circ} \mathrm{C}$, com fotoperíodo de 12 horas de luz branca. A condição de escuro foi obtida com o envolvimento das caixas Gerbox em três sacos de polietileno preto, sendo os experimentos montados e o acompanhamento feito em câmara escura, com luz verde de segurança. Os experimentos foram conduzidos por três meses e a contagem de sementes realizada semanalmente. As médias de cada tratamento foram transformadas pelo arcsen . proporção ${ }^{1 / 2}$ antes das diferenças serem avaliadas pelo teste $\mathrm{t}$ de Student, para $\alpha=0,05$ (Sokal \& Rohlf 1981).

Germinação em solo inundado - Três grupos de 100 sementes cada foram colocados para germinar separadamente em caixas d'água contendo solo coletado na floresta higrófila, mantidos sob sombrite e irrigados diariamente (CC). Outras 300 sementes foram semeadas nas mesmas condições, no entanto foram mantidas parcialmente enterradas, com água a $2 \mathrm{~cm}$ acima do nível do solo e constantemente renovada (AC). Neste caso, um fluxo lento era mantido pela entrada e saída da água, através de canos de PVC. Após 60 dias neste local, 100 sementes foram retiradas e colocadas em solo na capacidade de campo $(\mathrm{AC}+\mathrm{CC})$; outras 100 retiradas, escarificadas e novamente semeadas em solo inundado (AC+E) e as 100 últimas, mantidas no mesmo tratamento com água corrente (AC). $\mathrm{O}$ experimento foi conduzido durante quatro meses e a germinação avaliada a cada 15 dias.

Crescimento de plântulas em solo inundado - Sementes de $C$. brasiliense foram semeadas em areia e as mudas transferidas para sacos de polietileno contendo areia grossa e fina (1:3). Após três meses, 90 plantas foram divididas em três grupos mantidos em solo na capacidade de campo (CC), solo inundado com água parada (AP) e com água corrente (AC). Os dois últimos grupos foram colocados em caixas d'água e submetidos à inundação da mesma forma descrita para o experimento com sementes. No tratamento AP houve renovação quinzenal da água a fim de diminuir o efeito do acúmulo de toxinas. A inundação teve a duração de 90 dias, momento em que as plantas inundadas foram retiradas dos tanques e mantidas em solo na capacidade de campo por mais 60 dias. No início do experimento, e a intervalos de 30 dias, seis plantas de cada tratamento foram coletadas para se medir o comprimento e determinar a massa da matéria seca (parte aérea e raiz) que foi obtida após secagem do material em estufa a $80^{\circ} \mathrm{C}$. Modificações morfológicas apresentadas pelas plantas durante o experimento foram anotadas. As médias de cada tratamento foram comparadas por análise de variância (ANOVA), e as diferenças comprovadas por um teste d.m.s., para $\alpha=0,05$ (Sokal \& Rohlf 1981). 


\section{Resultados}

Germinação - As sementes de C. brasiliense em capacidade de campo germinaram lentamente, tanto no experimento em laboratório quanto em viveiro. A semeadura em solo de floresta apresentou melhores resultados que em areia esterilizada pois, após 12 semanas, a germinação em laboratório era inferior a $50 \%$ (Fig. 1A), enquanto no viveiro atingia $76,3 \%$ (Fig. 1B).

As taxas de germinação no claro e no escuro foram praticamente iguais durante todo o experimento ( $\mathrm{t} \leq 2, \mathrm{P} \geq 0,08$ ), indicando que as sementes de $C$. brasiliense são indiferentes à luz (Fig. 1A).

A saturação hídrica do solo inibe a germinação. Sementes que foram mantidas nestas condições durante todo o experimento não germinaram (AC), e as que foram transferidas para solo drenado $(\mathrm{AC}+\mathrm{CC})$ não diferiram do controle (CC). A escarificação de sementes mantidas em solo hidricamente saturado $(\mathrm{AC}+\mathrm{E})$ não alterou a germinação (Fig. 1B).
Tolerância de plântulas à saturação hídrica do solo - Após dez dias de tratamento, algumas plantas inundadas com água corrente (AC) e água parada (AP) apresentaram algumas lenticelas hipertróficas acima e abaixo do nível de água. Raízes adventícias foram raras e surgiram em apenas uma planta do tratamento AP. Senescência e abscisão foliares não foram observadas. Durante a submersão, as raízes foram deteriorando-se e sendo substituídas por novas.

C. brasiliense apresentou crescimento lento em todos os tratamentos, mesmo nas plantas controle. A massa da matéria seca da parte aérea e da raiz de plantas submetidas à inundação não diferiu do controle em nenhum momento durante o experimento ( $\mathrm{F} \leq 2,01, \mathrm{GL}=2, \mathrm{P}>0,05)$. $\mathrm{O}$ aumento de massa da matéria seca das plantas que foram retiradas dos tanques foi a mesma das mantidas em capacidade de campo (Fig. 2).

A manutenção do crescimento das plantas inundadas foi confirmada pelas medidas de comprimento (Fig. 3). A parte aérea de plantas mantidas em capacidade de campo cresceu tanto quanto a de plantas inundadas $(\mathrm{F} \leq 2,41$,

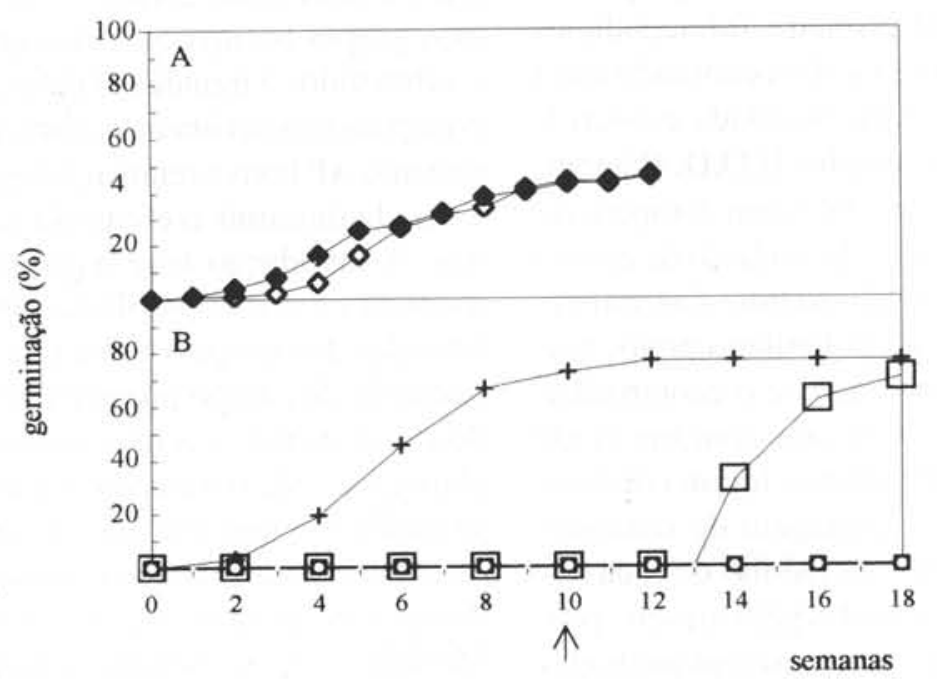

Figura 1. Germinação de sementes de Calophyllum brasiliense, em laboratório, sob diferentes condições de luz (A), e em viveiro, submetidas a regimes hídricos distintos (B). Tratamentos: A) - - - claro, - - escuro; B) -- solo na capacidade de campo (CC), - $\mathbf{E}$ - solo inundado (AC), $-\square$ - solo inundado por 10 semanas e posteriormente na capacidade de campo $(\mathrm{AC}+\mathrm{CC})$, $-\mathrm{O}$ - solo inundado por 10 semanas e posteriormente sementes escarificadas e colocadas em solo inundado $(\mathrm{AC}+\mathrm{E})$. A seta indica o momento da mudança nos tratamentos $\mathrm{AC}+\mathrm{CC}$ e $\mathrm{AC}+\mathrm{E}$. 


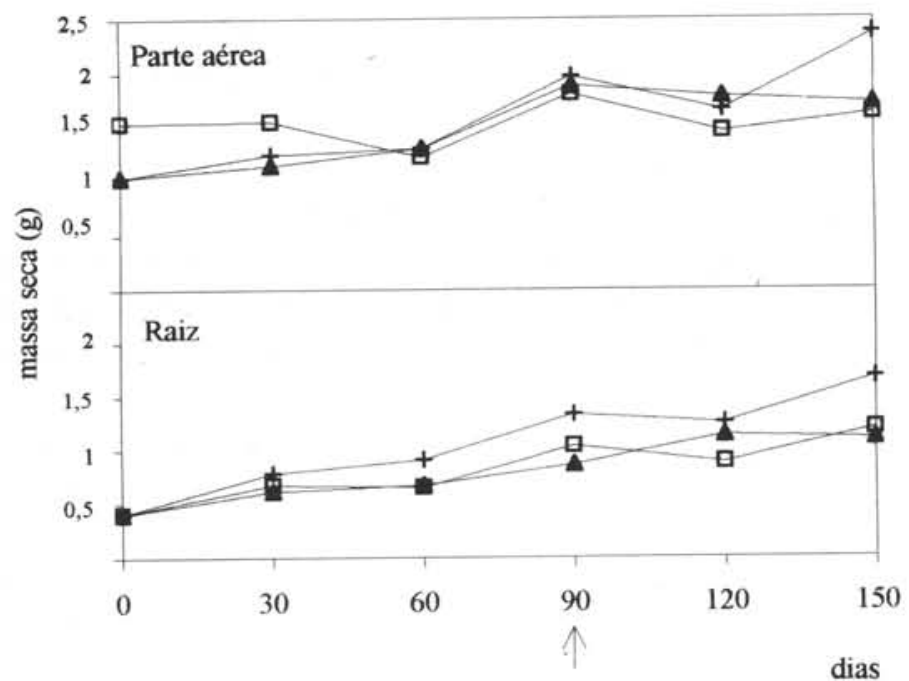

Figura 2. Massa da matéria seca da parte aérea e da raiz de plântulas de $\boldsymbol{C}$. brasiliense, em viveiro, submetidas a

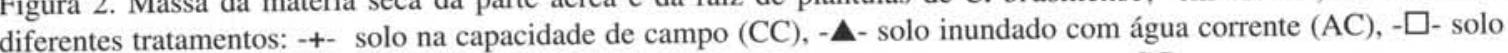
inundado com água parada (AP). A seta indica a mudança dos tratamentos AC e AP para CC.

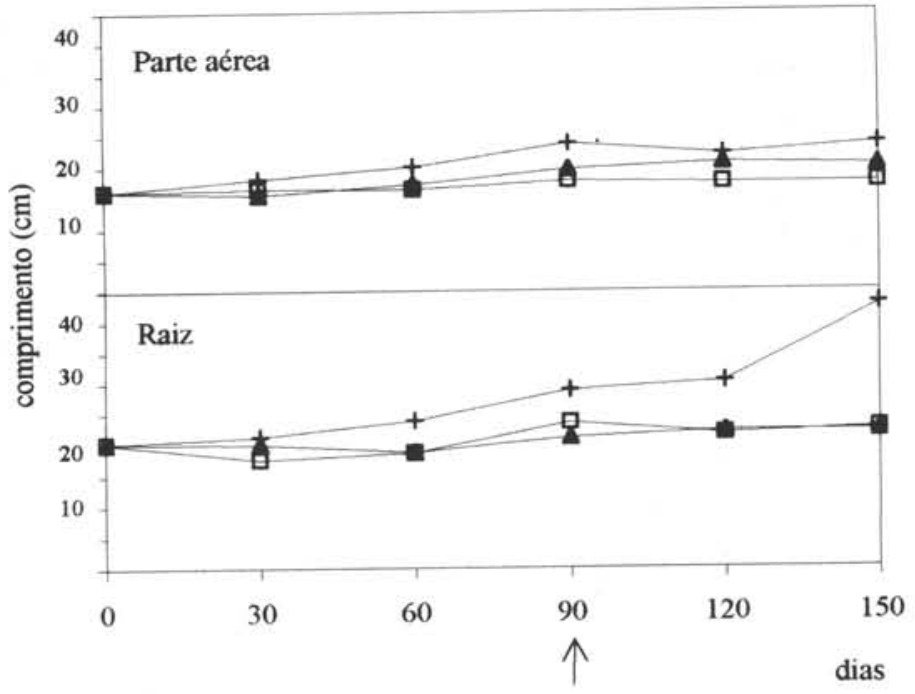

Figura 3. Comprimento da parte aérea e da raiz de plântulas de $\boldsymbol{C}$. brasiliense, em viveiro, submetidas a diferentes tratamentos: -+- solo na capacidade de campo (CC), - $\mathbf{\Delta}$ - solo inundado com água corrente (AC), $-\square$ - solo inundado com água parada (AP). A seta indica a mudança dos tratamentos AC e AP para CC.

$\mathrm{GL}=2, \mathrm{P}>0,05)$. Durante todo o experimento, os comprimentos das raízes de plantas dos três tratamentos também foram iguais $(\mathrm{F} \leq 3,6, \mathrm{GL}=2$, $\mathrm{P}>0,05)$, mas, após 150 dias, houve diferença entre o crescimento de raízes inundadas e não inundadas $(\mathrm{F}=11,63, \mathrm{GL}=2$, d.m.s. $=16,48$, $\mathrm{P}<0,05)$.

\section{Discussão}

O sucesso no estabelecimento de uma espécie em áreas inundadas depende, inicialmente, da estratégia de frutificação e dispersão dos frutos, associada à capacidade desses sobreviverem na água. Os frutos de $C$. brasiliense são 
dispersos por morcegos e pela água, no período mais chuvoso (Marques \& Fischer, dados não publicados). O solo da floresta higrófila, que normalmente apresenta um mosaico de condições hídricas distintas (Joly 1986), está encharcado no verão. Apesar da semente não germinar nestas condições ela se mantém viável, pelo menos por três meses, submersa ou enterrada no solo encharcado. Ao encontrar microsítios favoráveis, seja devido ao abaixamento do nível do lençol freático ou à chegada a um local de solo mais drenado, a semente germina.

Esta estratégia difere daquela apresentada por outras espécies típicas de ambientes sazonal ou permanentemente encharcados. No caso de Talauma ovata St.-Hil., que também é encontrada em florestas higrófilas, a dispersão das sementes ocorre no período seco e as sementes não só deixam de germinar, como perdem a viabilidade se mantidas submersas (Lobo \& Joly 1996; Lobo-Faria 1998). Por outro lado, espécies do gênero Inga, como Inga vera Willd. subsp. affinis T. D. Penn. (Lieberg \& Joly 1993; Okamoto 1998) e Inga laurina (Swartz) Willd. (Okamoto 1998), típicas de ambientes sujeitos à saturação hídrica do solo, frutificam em plena estação chuvosa e a submersão não inibe o potencial nem a velocidade de germinação. Até mesmo as sementes de Inga sessilis (Vel.) Mart., espécie típica de florestas com solos bem drenados, são capazes de germinar submersas, mas neste caso a taxa de germinação não ultrapassa os $40 \%$ (J. M. Okamoto \& C. A. Joly, dados não publicados). No outro extremo, encontram-se as espécies que não toleram a saturação hídrica do solo, para as quais apenas dez dias de submersão são suficientes para inibir completamente a germinação (Marques et al. 1992).

A ausência de germinação em sementes de C. brasiliense escarificadas e submersas reforça a idéia de que a inibição da germinação não se deve à impermeabilidade de tegumentos, mas sim a algum processo endógeno envolvido no acúmulo de toxinas (Crawford 1977) ou a al- gum tipo de dormência (Scarano et al. 1997). Mesmo em solo drenado as sementes apresentam níveis variáveis de dormência pós-dispersão pois a germinação não foi sincrônica e ocorreu gradativamente ao longo de 12 semanas (Fig. 1A e 1B). É provável que o endocarpo e a testa ofereçam alguma resistência, já que a germinação sem estas duas estruturas é mais rápida e eficiente (observações pessoais).

A indiferença ao estímulo fotoblástico somada ao fato da espécie não formar banco de sementes no solo, das plântulas serem pouco sensíveis às oscilações no regime de radiação e terem crescimento lento (Marques 1994) sugere que $C$. brasiliense seja uma espécie dos estádios finais da sucessão (Swaine \& Whitmore 1988). Sementes de espécies de florestas ciliares como $C$. brasiliense, que não apresentam exigência quanto à luz e ainda toleram a hipoxia, levam vantagem na ocupação de ambientes ribeirinhos, onde a previsibilidade ambiental é baixa.

Outro aspecto importante para a sobrevivência de uma espécie em ambiente sujeito a inundações refere-se às adaptações morfoanatômicas da planta à baixa disponibilidade de oxigênio no substrato. Em plântulas de $C$. brasiliense experimentalmente submetidas à inundação foi observada baixa quantidade de lenticelas hipertróficas e de raízes adventícias, que poderiam permitir aumento na difusão de oxigênio da parte aérea para o sistema radicular (Joly 1996; Marques et al. 1996). Observações de campo permitem afirmar que, em condições naturais, estas estruturas também são pouco freqüentes, embora seja possível a existência de aerênquima, mesmo antes da inundação, que auxilie na difusão de oxigênio em condições hipóxicas.

A tolerância à hipoxia torna-se ainda mais evidente com a capacidade das plantas jovens de $C$. brasiliense crescerem normalmente quando submetidas a esta situação. $C$. brasiliense, que em condições de arejamento do solo tem crescimento lento, cresceu normalmente duran- 
te o experimento de saturação hídrica do solo. Mesmo no tratamento com água parada, condição mais extrema de hipoxia, as plântulas mantiveram aumento da biomassa similar ao controle. A deterioração da raiz original em plantas inundadas tornou o seu comprimento inferior ao das plantas controle (Fig. 3), mas a produção de novas raízes em substituição às primeiras, foi eficaz, tanto que a massa da matéria seca permaneceu igual ao das plantas mantidas em capacidade de campo. As novas raízes que se desenvolveram no solo encharcado eram mais curtas, mais espessas e pouco ramificadas, semelhantes às observadas em raízes de outras plantas tolerantes a inundação (Lobo \& Joly 1998). Estas raízes apresentam, geralmente, aerênquima bem desenvolvido, o que facilita a aeração e a manutenção de metabolismo respiratório pelo menos parcialmente aeróbico, representando importante estratégia para a sobrevivência de plantas em áreas inundadas.

Em situação experimental, espécies que ocorrem em solos inundáveis geralmente apresentam este tipo de resposta, e espécies intolerantes a esta situação têm inibição do crescimento já na fase de germinação da semente (ver exemplos em Lobo \& Joly 1998). Paradoxalmente, na espécie amazônica Parkia pendula (Willd.) Benth. ex Walp., apesar das sementes sobreviverem após sete meses de submersão, as plântulas não toleram esta condição e morrem após poucas semanas, justificando a ocorrência da espécie somente em áreas marginais (Scarano \& Crawford 1992). Estes exemplos demonstram a diversidade de estratégias que as plantas tropicais apresentam quando submetidas à inundação.

A capacidade das sementes manterem a viabilidade submersas e das plantas crescerem normalmente em solo encharcado mostram que $C$. brasiliense é espécie para a qual a saturação hídrica do solo não interfere de forma negativa em seu ciclo de vida. Estas características associadas à diversidade de mecanismos de dispersão, justificam a ampla distribuição da espécie, que contribui significativamente para a estrutura e a fisionomia das florestas que ocorrem em áreas sujeitas a inundações, desde a América Central até o litoral de Santa Catarina (Reitz et al. 1978).

Os resultados encontrados demonstram que C. brasiliense possui um conjunto de características que permitem que seja recomendada para a recomposição da vegetação nativa (Joly et al. 1999) e o manejo de áreas marginais a cursos d'água, como tem sido realizado em alguns reservatórios de usinas hidrelétricas no Estado de São Paulo (Salvador et al. 1992).

\section{Agradecimentos}

Os autores agradecem à Dra. Katia Christina Zuffellato Ribas pela leitura do manuscrito; ao Parque Ecológico da UNICAMP, pelo cultivo das mudas; ao CNPq pela cessão de bolsa à primeira Autora e às instituições financiadoras do projeto: Prefeitura Municipal de Brotas (SP), Consórcio Intermunicipal para a Preservação da Bacia do Jacaré-Pepira, IBAMA (Instituto Brasileiro do Meio Ambiente e Desenvolvimento), CNPq, FINEP, FAPESP, FAEP/UNICAMP, Conservation International, UNESCO/MAB e Grupo Pão de Açúcar.

\section{Referências bibliográficas}

Crawford, R. M. M. 1977. Tolerance of anoxia and ethanol metabolism in germinating seeds. New Phytologist 79: 511-517.

Crawford, R. M. M. \& Brändle, R. 1996. Oxygen deprivation stress in a changing environment. Journal of Experimental Botany 47: 145-159.

Hook, D. D. 1984. Adaptation to flooding with fresh water. Pp. 265-294. In: T. T. Kozlowski (Ed.), Flooding and plant growth. Academic Press, London.

Joly, C. A. 1986. Heterogeneidade ambiental e diversidade de estratégias adaptativas de espécies arbóreas de mata de galeria. Pp. 19-38. In: Anais do X Simpósio anual da ACIESP, São Paulo.

Joly, C. A. 1991. Flooding tolerance in tropical trees. Pp. 23-24. In: M. B. Jackson, D. D. Davies \& H. Lambers (Eds.), Plant life under oxygen deprivation: ecology, physiology and biochemistry. SBP Academic Publishing, The Hague. 
Joly, C. A. 1996. The role of oxygen diffusion to the root system on the flooding tolerance of Brazilian trees. Revista Brasileira de Biologia 56(2): 375-382.

Joly, C. A.; Spigolon, J. R.; Lieberg, S. A.; Aidar, M. P. M.; Salis, S. M.; Metzger, J. P. W.; Zickel, C. S.; Lobo, P. C.; Shimabukuro, M. T.; Salino, A. \& Marques, M C. M. 1999. Projeto Jacaré-Pepira - os resultados do desenvolvimento de um modelo de recomposição da vegetação do ecótono ciliar com base na florística regional. In: R. R. Rodrigues (Ed.), Matas ciliares: estado atual do conhecimento. Ed. da Universidade Estadual de Campinas, Campinas (no prelo).

Kozlowski, T. T. 1984. Responses of woody plants to flooding. Pp. 129-163. In: T. T. Kozlowski (Ed.), Flooding and plant growth. Academic Press, London.

Lieberg, S. A. \& Joly, C. A. 1993. Inga affinis DC (Mimosaceae): germinação e tolerância de plântulas à submersão. Revista Brasileira de Botânica 16(2): 175-179.

Lobo-Faria, P. C. 1998. Estratégias adaptativas de espécies arbóreas típicas de ambientes de solo hidricamente saturado: uma abordagem morfológica, bioquímica e ecofisiológica. Tese de Doutorado. Universidade Estadual de Campinas, Campinas.

Lobo, P. C. \& Joly, C. A. 1996. Ecofisiologia da germinação de sementes de Talauma ovata St.-Hil. (Magnoliaceae), uma espécie típica de matas de brejo. Revista Brasileira de Botânica 19(1): 35-40.

Lobo, P. C. \& Joly, C. A. 1998. Tolerance to hypoxia and anoxia in neotropical tree species. Oecologia Brasiliensis 4: 137-156.

Marques, M. C. M. 1994. Estudos auto-ecológicos do guanandi (Calophyllum brasiliense Camb. Clusiaceae) em uma mata ciliar do município de Brotas, SP. Dissertação de Mestrado. Universidade Estadual de Campinas, Campinas.

Marques, M. C. M.; Pimenta, J. A. \& Colli, S. 1992. Germinação de Cedrela fissilis Vell. e Parapiptadenia rigida (Benth) Bren. após pré-tratamento em condições hipóxicas e posterior estocagem a seco. Revista do Instituto Florestal 4: 620-624.

Marques, M. C. M.; Pimenta, J. A. \& Colli, S. 1996. Aspectos do metabolismo e da morfologia de Cedrela fissilis Vell. e Anadenanthera colubrina (Vell.) Bren. submetidas a diferentes regimes hídricos. Arquivos de Biologia e Tecnologia 39(2): 385-392.

Oliveira-Filho, A. T. \& Ratter, J. A. 1995. A study of the origin of central Brazilian forests by the analysis of plant species distribution patterns. Edinburg Journal of Botany 52(2): 141-194.
Okamoto, J. M. 1998. Ecofisiologia da germinação e do metabolismo respiratório de quatro espécies do gênero Inga Mill. (Mimosaceae) submetidas à hipoxia e anoxia. Dissertação de Mestrado. Universidade Estadual de Campinas, Campinas.

Ponnamperuma, F. N. 1984. Effects of flooding on soil. Pp. 10-43. In: T. T. Kozlowski (Ed.), Flooding and plant growth. Academic Press, London.

Reitz, R.; Klein, R. M. \& Reis, A. 1978. Projeto madeira de Santa Catarina. Sellowia 28-30.

Rodrigues, R. R. 1989. Análise estrutural de formações ripárias. Pp. 99-119. In: L. M. Barbosa (Ed.), Anais do Simpósio sobre mata ciliar. Fundação Cargil, Campinas.

Salvador, J. L. G. 1987. Considerações sobre matas ciliares e a implantação de reflorestamentos mistos nas margens de rios e reservatórios. Série Divulgação e Informação, 105. CESP, São Paulo.

Salvador, J. L. G.; Oliveira, S. B.; Oliveira, D. B. \& Silva, J. R. 1992. Comportamento do guanandi (Calophyllum brasiliense) em solos úmidos, periodicamente inundáveis e brejosos. P. 24. In: P. Y. Kageyama (Ed.), Recomposição da vegetação com espécies arbóreas nativas em reservatórios de usinas hidrelétricas da CESP. IPEF, Piracicaba.

Scarano, F. R. 1998. A comparison of dispersal, germination and establishment of wood plants subjected to distinct floodings regimes in Brazil floodprone forests and estuarine vegetation. Pp. 177-194. In: F. R. Scarano \& A. C. Franco (Eds.), Ecophysiological strategies of xerophytic and amphibious plants in the neotropics. Series Oecologia brasiliensis vol. IV. PPGE-UFRJ, Rio de Janeiro.

Scarano, F. R. \& Crawford, R. M. M. 1992. Ontogeny and the concept of anoxia-tolerance: the case of Amazonian leguminous tree Parkia pendula. Journal of Tropical Ecology 8: $349-352$.

Scarano, F. R.; Ribeiro, K. T.; Moraes, L. F. D. \& Lima, H C. 1997. Plant establishment on flooded and unflooded patches of a freshwater swamp forest in southeastern Brazil. Journal of Tropical Ecology 14: 793-803.

Sokal, R. R. \& Rohlf, J. E. 1981. Biometry. Freeman, San Francisco.

Swaine, M. D. \& Whitmore, T. C. 1988. On the difinition of ecological species groups in tropical rain forests. Vegetatio 75: 81-86. 